\title{
Affective Consumer Requirements: A Case Study of Moisturizer Packaging
}

\author{
Brian Henson, ${ }^{1, *}$ Cathy Barnes, ${ }^{1}$ Rebecca Livesey, ${ }^{1}$ Tom Childs ${ }^{1}$ and Keith Ewart ${ }^{2}$ \\ ${ }^{1}$ School of Mechanical Engineering, University of Leeds, Leeds, LS2 9JT, UK \\ ${ }^{2}$ Procter and Gamble Technical Centres
}

\begin{abstract}
Tools for eliciting and managing product requirements are now well-established in some fields of engineering. These tools primarily focus on linking objective, functional customer requirements to the product's properties. Whilst there have been advances in identifying human factors requirements, the elicitation of the customers' subjective requirements of a product remains a challenge. This article reports a comprehensive case study in the use of affective engineering to elicit the subjective requirements for moisturizer packaging. The methodology uses focus groups and surveys to elicit subjective requirements. The results of semantic questionnaires are reduced using principal components analysis to translate the subjective requirements into values for physical properties of the packaging. The resulting requirements for surface textures, shape, and color were validated using questionnaire responses to prototype packaging. The study highlights research issues associated with recombining stimuli that have been tested separately.
\end{abstract}

Key Words: Kansei engineering, affective engineering, human factors design, customer requirements.

\section{Introduction}

Tools for eliciting and managing product requirements are now well established in some fields of engineering [1-3], to the extent that safety critical systems, for example, are often verified using formal languages. These tools primarily focus on linking the functional customer requirements to the product's properties. While there have been advances in identifying human factors requirements, such as ergonomics and usability, the elicitation of the customers' subjective requirements of a product, such as feeling pleasure when using the product, remains a challenge. And yet, as many products mature in the marketplace, consumers' expectations have evolved such that functionality and usability are taken for granted, and the remaining product differentiators are the subjective responses to the product experienced by the customer. This article reports a study into the use of affective engineering to elicit the subjective requirements for moisturizer packaging.

Affective engineering is a relatively new field of design concerned with understanding people holistically,

*Author to whom correspondence should be addressed.

E-mail: b.henson@leeds.ac.uk

Figures 2-7 appear in color online: http://cer.sagepub.com judging the superiority of the design on the basis of a broader relationship between the product and the individuals for whom it was designed [4]. Affective engineering involves translating consumers' feelings for a product into design elements. Affective engineering is closely related to Kansei Engineering, which has been developed since the 1970s by Professor Nagamachi of Hiroshima University. Kansei Engineering has been successfully applied to many products, such as car interiors and cosmetics. See [5] for a full review. There are different manifestations of Kansei $[6,7]$.

The noun 'affect' refers to several psychological states such as emotions, feelings, moods, sentiments, and passions, each of which differs in duration and impact. This study is principally concerned with the emotions and feelings elicited by product packaging and also considers subjective responses that are judgments, such as whether a product looks expensive. To avoid ambiguity, the words 'subjective response' are used in this article when referring to affective responses and subjective judgments.

While there are a number of tools available for eliciting subjective requirements that are complementary to affective engineering (such as experience-based design [8], product personality profiling [9], ethnography [10], storyboards [11], and product semantics [12]), affective engineering attempts to relate the subjective requirements to measurable product properties that can 


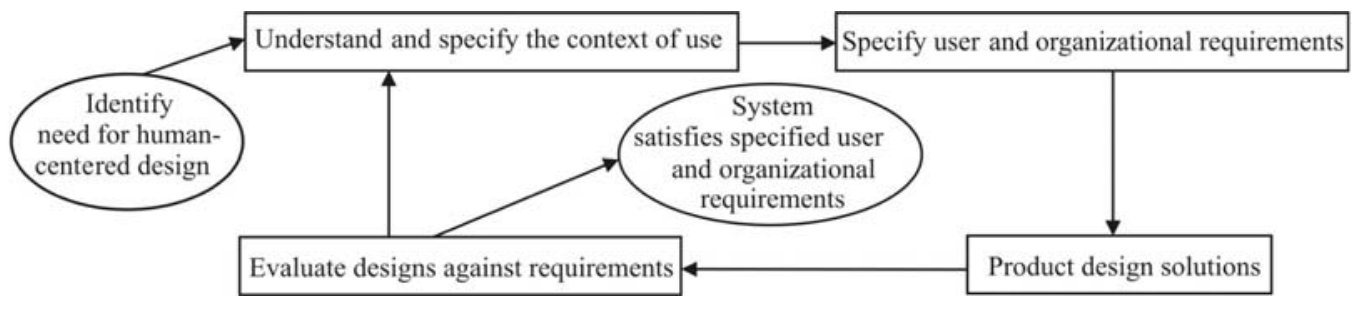

Figure 1. Human-centered design process from ISO 13407 [13].

be tested and verified. This principle is illustrated by considering affective design as a set of tools with which to populate the human-centered design process from ISO 13407 [13] (Figure 1). The principle of affective design is highlighted by the tasks to specify the context of use and user requirements (in this case the subjective) and to later evaluate the designs against those requirements. This differs from many traditional processes in which the design evolves based on the experience and creativity of the designer and denies the possibility of an objective assessment of the final design against the stated specification.

The steps of the methodology used in this study, which map onto the phases of the human-centered design process, are as follows:

1. Identify product context. The first step is to develop an understanding of the product, packaging, consumer, and market contexts using techniques such as focus groups and video ethnography, and hence to identify the product features that have the most influence on the subjective requirements of consumers. The motivation for this study came from Procter and Gamble, who wanted to develop the packaging for a body moisturizer as a brand extension to an existing product range. The target demographic for the study was women aged between 18 and 50 who already purchased one of the company's products. In this study, the focus group was used to understand and specify the product context.

2. Adjective generation and reduction. Step two is to use the understanding of the product context to develop a corpus of adjective pairs that can be used to assess the required subjective response. The list of pairs is reduced to 20 or so using pilot studies or affinity diagrams for use in a semantic differential questionnaire. In this study, focus groups were used to generate adjectives and a pilot questionnaire was used to reduce them.

3. Create test stimuli. Stimuli are generated using the output from the step 2. In this study, the number of stimuli was reduced using semantic mapping.

4. Perform semantic differential experiment. The approach taken in this study was to use the semantic differential technique described by Osgood et al. [14].
5. Analyze and interpret results. The data from step 5 is reduced using principal components analysis and component scores are calculated for the stimuli. This allows the placement of the stimuli in perceptual space to support an interpretation of their subjective properties.

6. New product evaluation. The results from step 6 are used to identify the subjective requirements for a new product. A prototype of the new product is created with which to validate the results of the study.

In the remainder of this article, a case study using this affective engineering methodology is presented.

\section{Identify Product Context and Collect Adjectives}

Demographic information was collected using a questionnaire distributed to 25 females. The questionnaire asked about age, levels of wealth (inferred from postcodes) and their moisturizer purchasing habits. This information allowed the identification of users who fit the demographic who could then be recruited for further studies.

The shapes, volumes, textures, and colors of existing packaging were analyzed to provide an understanding of current products.

Nine female participants from the specified demographic were recruited to a focus group. All the focus groups in this study conformed to the format described by Krueger and Casey [15]. The purpose of the focus group, which was not disclosed to the participants, was to gather information about brand recognition and consumer feelings about packaging, and to collect adjectives for the semantic study. The focus group meeting took place in comfortable surroundings, around a round table, was moderated by a female, and was video recorded. First, the participants were asked to offer names of different skincare brands or products. Second, the group was asked to observe pictures of merchandise on shop shelves and asked which product or brand was more noticeable and why. Third, the group ranked brands according to their preference taking into account product quality and packaging. Fourth, the participants were asked to select 
a moisturizer that appealed to them and were encouraged to try it. Participants were then asked individually why they chose that particular product and what they liked and disliked about it. Finally, participants were asked which adjectives they would use to describe moisturizers. Stimulation was provided by displaying a slide show of magazine adverts for moisturizers. The adjectives used by the focus group were supplemented by synonyms and words from beauty magazines and advertisements.

Of the 25 women who took part in the first demographic survey, 11 used the Procter and Gamble brand. Of those, all owned their own property, whereas only $25 \%$ of those who used other brands owned their own home. The youngest of the users of the Procter and Gamble brand was 38 years old. The findings were consistent with information about the target demographic provided by Procter and Gamble.

During the focus group study, brand recognition repeatedly arose as a reason for mentioning a product. When shown pictures of shop displays of moisturizers, many participants recognized particular brands through their shape, graphic design, or colors. Selecting a product because it is recognizable is a theme that continued to emerge throughout the study.

Packaging design also influenced people's choice. One participant said, 'I think it's probably the shape and the color of the packaging', when asked why she had identified a particular moisturizer from a picture. One said 'The way it's packaged and put on the shelf' can influence their purchase decision, and another said, 'The packaging for me was too loud'.

Participants' shape preferences appeared to revolve around basic shapes, such as tubs or jars, and getting value for money. One said of tubs 'you're spending less on packaging so you feel you're getting more value for money'. The lighter colors of packaging seemed more popular, generating adjectives such as 'pure', light greens were thought 'fresh', whereas the 'loud' orange or yellow packaging were found to be a put-off.

Cost was frequently mentioned. For example, color can make consumers feel 'as though they probably cost a bit more'. The shape of the packaging can convey an image of luxury, or conversely simplicity and hence value for money. The shape of the product affects how much is left at the bottle of the jar. Paler colors created a greater sense of value, giving the impression less was being spent on the packaging. Complex pump mechanisms were assumed to be a large part of the final cost and participants felt this was a waste of money. Ease of use was also mentioned frequently during the focus group.

How the products felt when applied to the skin was discussed in the focus group, although the texture of the packaging was not mentioned. When prompted by the facilitator, 'When you're buying a product do you ever tend to pick it up before you make the decision to buy it? Do you ever pick it up and have a feel?', all of the ladies replied, 'Oh yeah', 'Always'.

Showing advertisements for moisturizers to the participants of the focus group was an effective method for eliciting adjectives. For example, one magazine advertisement received a favorable response from the participants and generated words such as cosy, pure, contented, protecting, cold, and simple. These methods elicited 116 adjective pairs.

Although not an explicit issue amongst the participants in the focus group, both the researchers and Procter and Gamble thought surface textures of packaging materials to be an important issue during initial purchase decisions, and it was decided that investigation of textures should form part of the main study. Thus shape, color, and texture were chosen as the requirements of packaging to be investigated in the main study.

\section{Adjective Reduction}

The next stage of the study was to reduce the number of adjectives collected by the focus group to around 20 appropriate adjectives to be used in the main semantic differential experiment. The adjectives collected from the focus group were collated into a pilot semantic differential questionnaire accompanied by the antonym (i.e., happy and sad). The beginning of the questionnaire contained an introductory statement to explain the purpose of the survey and provide guidelines for answering the questions [16], and a pictorial representation of the scales were presented to aid understanding [14] (Figure 2).

The questionnaire comprised 116 adjective pairs. One pair (clean, dirty) was repeated three times to support reliability tests, and the adjectives were listed with random polarity [16]. The questionnaire was completed by 77 members of the target demographic.

To guarantee the adjective pairs chosen were dissimilar and cover all aspects of subjective responses towards the product, they were classified into evaluation, potency, and activity words [14]. The mean, standard deviation, and variance of the adjective pair scores were calculated. Those adjectives in each classification with the smallest variance, and hence the highest discrimination, were retained. The reliability of the questionnaire was tested using the test-retest method on those adjectives that had been repeated in the questionnaire. Reliability was measured using correlation coefficients (R-values) between the sets of responses. R-values were considered to be good if they exceeded 0.7 [17]. 


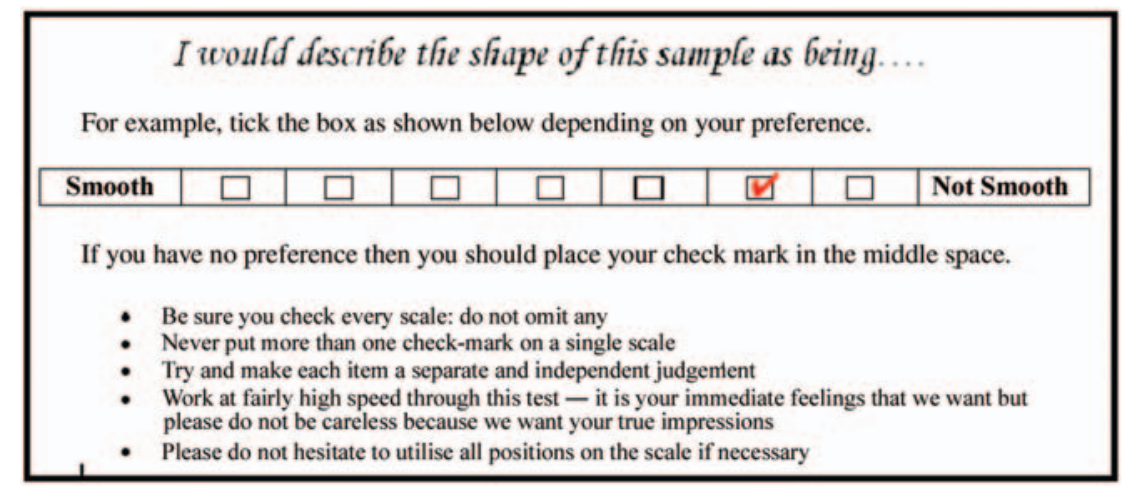

Figure 2. Header from a semantic questionnaire.
Four sets of respondents' data were removed because they exhibited high variance. The correlation coefficient of the remaining data was 0.76 , thus showing the survey reliable. The adjective reduction exercise resulted in the selection of thirteen adjective pairs. The adjective pairs were calming-not calming, velvety-rough, silky-not silky, relaxing-stressful, soft-hard, gentle-harsh, nourishing-unhealthy, soothing-irritating, clean-not clean, fresh-not fresh, revitalising-not revitalising, appealing-not appealing, and smooth-coarse.

\section{Stimuli Creation and Reduction}

Existing packaging shapes were classified according to volume, dimensions, proportions, and shape. $250 \mathrm{~mL}$ bottles were chosen as the stimuli as it is the size most associated with the higher-priced products that Procter and Gamble was interested in. The packaging shapes were classified as: cylinder downward taper, flask, tapered oval, teardrop (Figure 3), tube, upward taper, wide mouthed jar, and waisted.

The CAD models of 48 shapes based on the nine classifications were built. The models did not include features of the closure (such as caps or pumps) that might bias the respondents through functionality or usability issues. The CAD models were colored a neutral gray (N6 on the Munsell scale [18]). The CAD images of the shapes were displayed on a large screen and were set freely rotating to allow participants to view the models from all angles without having to touch keyboards.

To examine the effect of color, 72 rectangular panels were selected from the SCOTDIC Color Specifier system. Between six and nine panels were chosen from each of the five Munsell primary (red 5R, yellow 5Y, green $5 \mathrm{G}$, blue $5 \mathrm{~B}$, and purple $5 \mathrm{P}$ ) and five intermediate hue (yellow-red 5YR, green-yellow 5GY, blue-green 5BG, purple-blue 5PB, and red-purple 5RP) planes. The set was completed by three neutral panels. Each panel was surrounded by a neutral gray and presented to the participants in a color cabinet. The

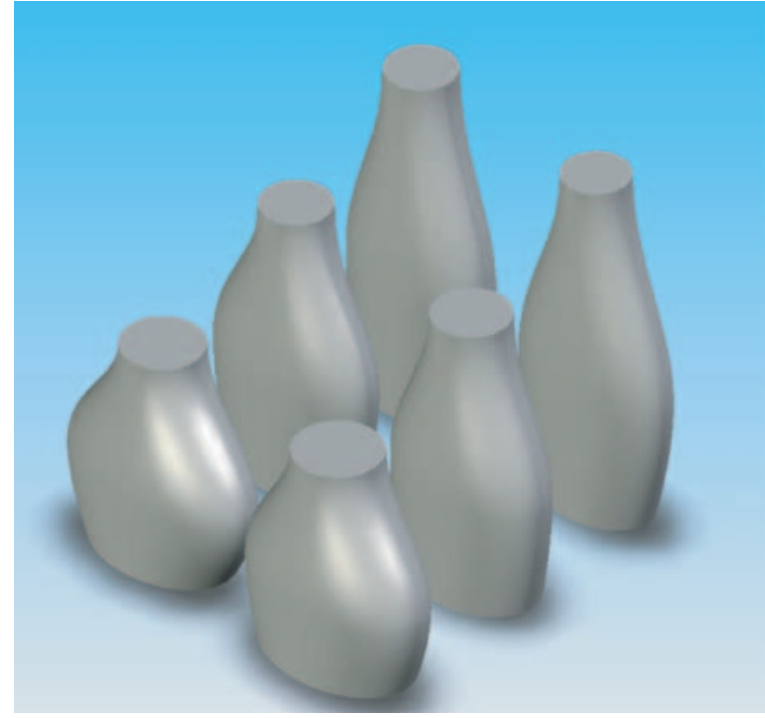

Figure 3. $C A D$ models of packaging in the teardrop category.

observers were seated a constant distance from the light cabinet throughout the experiment to ensure that all samples were viewed from the same angle.

The study of surface texture used existing materials from the packaging industry for the stimuli, such as plastics, paper, and card, plus some outliers in the form of textiles and fruit and vegetable skins. The stimuli were mounted on opposite sides of wooden blocks of dimensions $100 \times 45 \times 20 \mathrm{~mm}$ with the surface contained between card windows $(80 \times 25 \mathrm{~mm})$. The blocks were designed to be easily manipulated by the hands while touching the surfaces between thumb and forefinger and to ensure uniform stimulus size.

Fifty-five materials were collected and presented to participants on the blocks in large plain envelopes. Blind-folding was not used because it can lead to disorientation that influences emotional and cognitive functions [19]. Participants washed and dried their hands before taking part in the research. They were instructed to touch the stimuli in the way that was most natural to them. 
To minimize fatigue during the main experiment, semantic mapping was used to reduce the number of stimuli. Semantic mapping is a technique which assesses stimuli on a grid against two criteria. Thirty women from the chosen demographic were invited to attend one of the three sessions. Participants were encouraged to view and touch all stimuli before the study began. The participants were asked to place numbered counters representing each stimulus on a grid to indicate how much each stimulus scored against the axes. The axes had been labeled with the most important potency and activity adjectives from the adjective reduction exercise (Figure 4). The positions of the counters were noted for each participant and the coordinates averaged to produce a plot of stimulus position. The semantic grid technique was used to reduce the number of stimuli for the shape, color, and texture experiments.

Use of the semantic grid technique resulted in 13 shapes and 12 textures having the greatest association with the most desirable attributes of the product. The number of colors was reduced to ten, but four other colors were introduced based on the judgement of researchers to provide a more stimulating variety of colors.

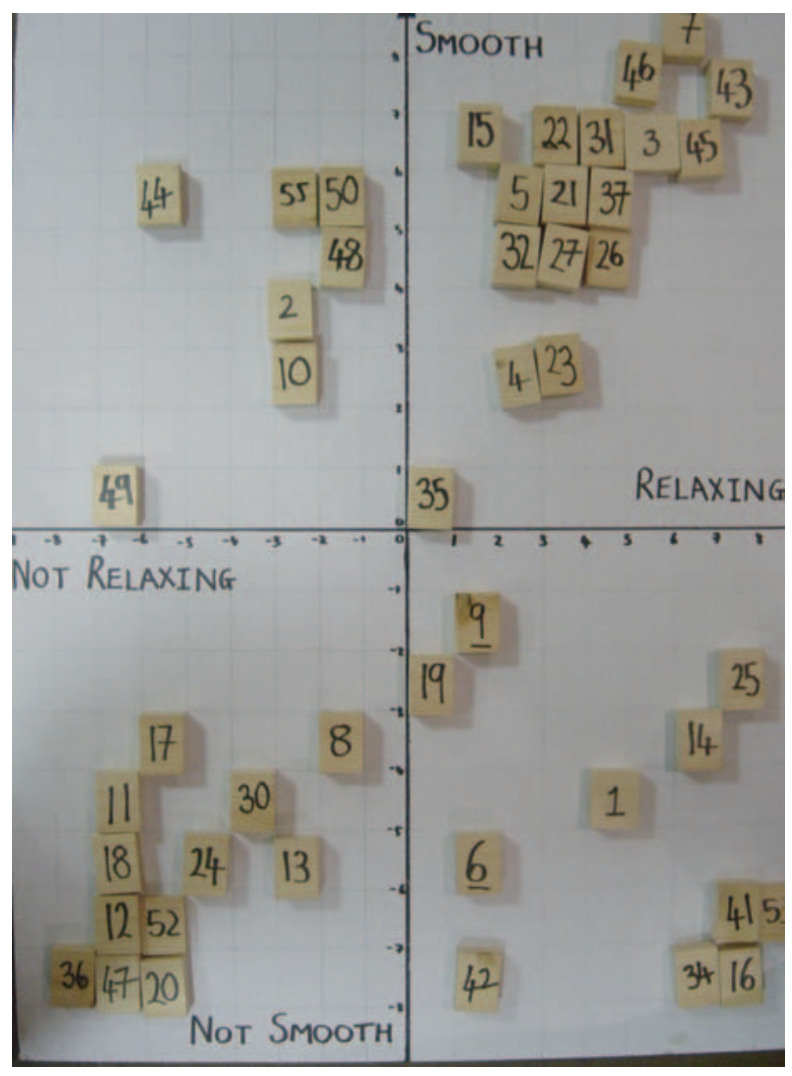

Figure 4. Counters placed on a semantic grid during a stimulus reduction exercise.

\section{Semantic Differential Experiment}

The semantic differential technique [14] was used to measure respondents' subjective responses to the shape, color, and texture stimuli. The format of the questionnaire used in the main experiment was similar to the adjective reduction questionnaire described in Section 3. The header of the questionnaire asked respondents to consider 'I would describe the shape/ texture/color of this sample as being...' and then check the box on the semantic scale that best described the stimulus. The questionnaire included a question that asked the participants for an opinion of the stimulus to provide clarity when analyzing the adjective ratings. For example, a participant may rate the stimulus as feeling not velvety but may consider this to be a positive characteristic. The presentation of stimuli and the order and polarity of the adjective pairs were randomized to reduce fatigue bias.

The questionnaire used in the main experiment differed from that in the adjective reduction exercise in that declarative opposites (clean, not clean), rather than antonyms (i.e., clean, dirty), were used. This was done at the request of Procter and Gamble.

According to rules set out by Bryant and Yarnold [20] the subjects-to-items ratio should be no lower than $5: 1$. As 13 adjective pairs were used, 65 people were recruited to take part. The respondents were presented with the stimuli using the various methods specified in the previous section and asked to complete semantic questionnaires. The results over all the studies were then analyzed.

A principal component analysis was carried out using SPSS version 11.0 to identify the factor loadings of the stimuli and hence determine the participants' subjective responses. The data input to the software was derived from the responses to the questionnaires. Each tick response was scored from -3 on the left to +3 on the right. For every adjective and stimulus the 65 responses were averaged. These were assembled as a matrix $X$ of order $n \times m$, where $n$ is the number of stimuli and $m$ the number of word pairs. Within the software, this was conditioned to standardized form $X_{S}$ (each of the $\mathrm{m}$ columns normalized to zero mean and unit standard deviation). The correlation matrix $X_{s}^{T} X_{s}$ of order $m \times m$ was created and from this the principal components were extracted using varimax rotation and Kaiser normalization. The output matrix $Z$ of word loadings on to the principal component directions was of order $m \times 2$. Finally, the $n \times 2$ matrix of item scores for shape, color, and texture in principal component space was obtained by the matrix multiplications of $X \cdot Z$.

The principal components analysis resulted in two factors with eigenvalues of greater than one which accounted for nearly $88 \%$ of the variance. The first component appeared to be dominated by words such 


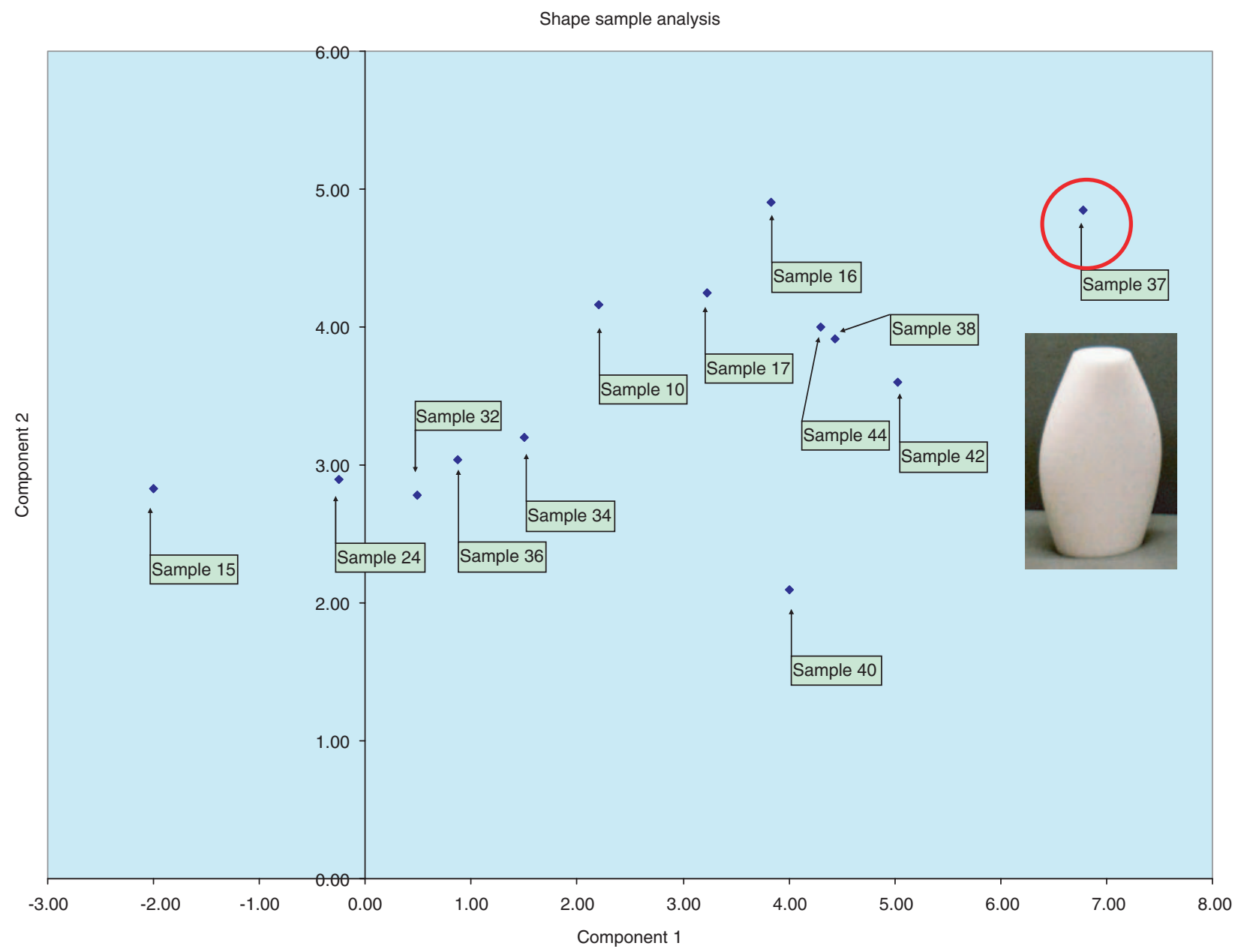

Figure 5. Component scores for shape stimuli.

as velvety and soft, and the second component was dominated by words such as fresh and revitalising. The item scores for each stimulus were plotted as graphs of component 1 against component 2. The ideal shape was found to be that classified as medium oval and the ideal color was 5P2 light heather (Figure 5). Velvet was found to have the highest component scores for surface texture, but due to difficulties of manufacturing packaging with velvet, the second highest scoring surface texture, a screen-printed soft-touch surface, was chosen.

\section{New-product Evaluation}

The final focus group was used to test whether the combination of the ideal color and shape identified by the main experiment would result in a packaging design preferred by those in the demographic. Due to the difficulties of manufacturing shapes with both the required color and surface texture, texture was not tested in this part of the experiment. This combination of color and shape was tested in two ways: through the use of PrEmo software [21] and a semantic questionnaire.

PrEmo is a software that uses animation and sounds, rather than adjectives, to describe emotions. In PrEmo, the respondent chooses the feelings elicited by a product by clicking 14 different pictures, each one representing a different emotion. Once the picture has been clicked the sound effect and animation is played and the respondent then clicks on a colored square depending on how strongly they feel this emotion towards the stimulus.

Six bottles for body moisturizer were painted different colors. The bottles were chosen so that each classification of packaging shape was represented. A medium oval shape was manufactured using a selective laser sintering machine. Two halves of a surface with the most favored texture were vacuumformed around the medium oval shape. The insides of the two halves were painted a color that closely matched 5P2 light heather, Dulux Himalayan Musk 5 (70RB 67/067), and the halves were glued to the laser-sintered core. The seven stimuli are shown in Figure 6. 

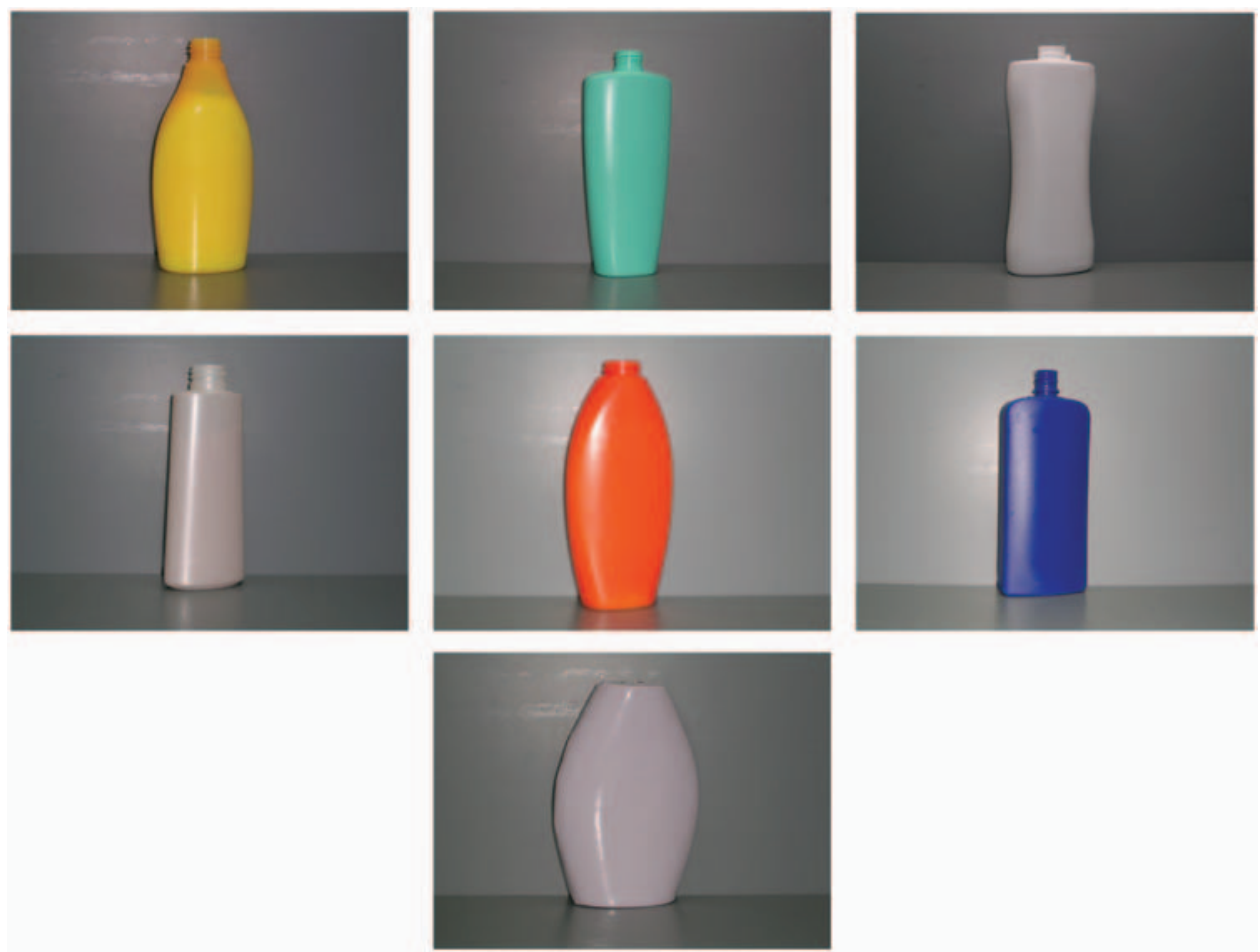

Figure 6. Stimuli for the validation study. The design incorporating the favorite shape and color is the one at the bottom of the picture.

Before the testing started, the participants were shown a screen containing pictures of all seven bottles, allowing the consumers to give a measured opinion during testing. Once the range of stimuli had been viewed, PrEmo was used to display pictures of each stimulus. Each respondent had their own computer and headset, and were able to view pictures of the stimuli and complete the tests in their own time. Ten people participated in this part of the study.

The final seven stimuli were also tested using a semantic questionnaire, the format of which was very similar to the previous two. The adjectives used were those 14 used in PrEmo so that direct comparisons between the results of the software and questionnaire could be made. In order to ensure consistency with the software, adjective quantifiers (very, somewhat, and neutral), which were not used on the previous semantic questionnaires, were introduced on the final one. The question at the beginning of the questionnaire was altered to read 'On viewing this bottle I feel...'. These changes were made at the request of Procter and Gamble.

The principal components of the data from the semantic questionnaire were identified and the component scores for each stimulus were calculated. The medium oval bottle in light heather scored highest against both components (Figure 7). Analysis of the data from PrEmo again revealed the medium oval bottle in light heather as the favorite, being placed close to emotions such as satisfaction, admiration, fascination, and desire. However, as the PrEmo and semantic instruments were being administered, it was noted that participants did not enjoy the surface texture of the prototype bottle.

\section{Discussion}

The first focus group clearly identified some of the subjective requirements of the packaging. As expected, shape and color were frequently mentioned but it was surprising that surface texture was not. The researchers found the focus groups to be beneficial when combined with the more structured experimental methods, because focus groups allowed insights that might otherwise have been missed, such as concerns about value for money and functionality.

The semantic grid technique was found to be an effective way to reduce the number of stimuli. However, problems arose because of fatigue bias. Another problem came from users placing counters on the basis of personal preference rather than whether they reflected well the adjectives on the axes. Furthermore, some people had trouble relating the various shapes, colors, or textures to the adjectives.

Manufacture of the final design assumed that combining the favorite shape, color, and surface texture would result in a package design that would also be the most favored. However, it is unlikely that stimuli can easily be combined in this way. For example, just because people like a particular color does not necessarily imply they will like it when it is combined with a 


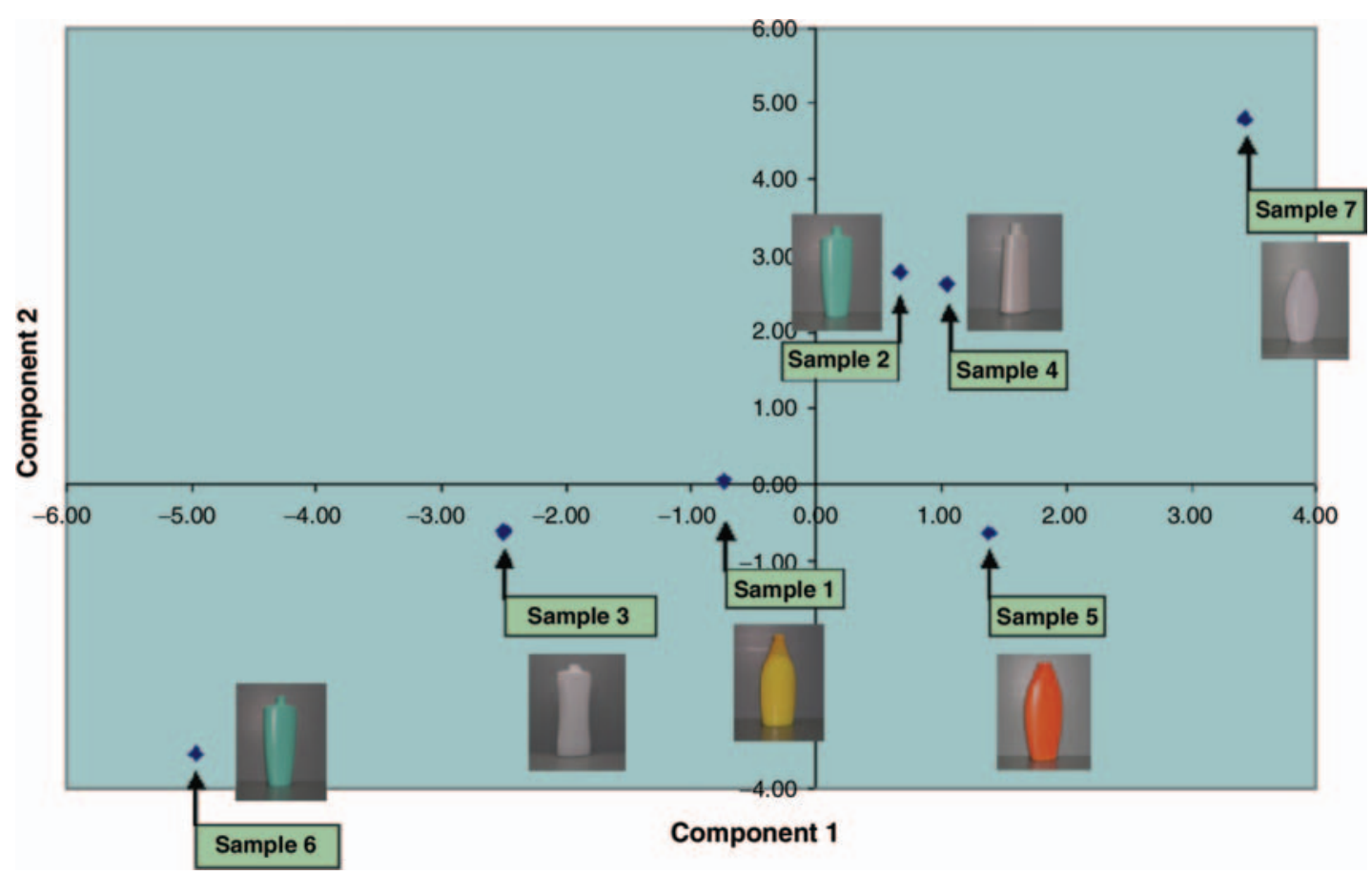

Figure 7. Component scores for stimuli in the validation experiment.

specific shape. It appears that although the combination of shape and color was successful, the surface texture was perceived to be incongruent. This could be because of the effect of visual perception of texture.

The literature suggests that how stimuli combine is not straightforward. Although many studies into perception have assumed that the brain analyses the color of a surface independently of its three-dimensional shape and viewing geometry, Bloj et al. [22] show color perception is strongly influenced by three-dimensional shape. On the other hand, Guest and Spence [23] report that, although multi-modal integration has been found in audiovisual, odor/taste and visuo-haptic size discrimination, 'providing congruent information in disparate modalities does not appear to enhance either perception or performance' in discrimination tasks. Thus, it is likely that how stimuli combine depends on which senses are used to perceive those stimuli. Another issue is whether stimuli scale well with each other; perception of color and texture could change with stimulus size.

Because of the need to isolate the subjective responses to shape, color, and surface texture, many other factors that could influence responses were ignored. For example, both smell and sound have been said to have an impact on color perception [24]. The functionality of the container was not included in the studies, although the initial focus group demonstrated that women found the dispensing method to be of utmost importance. Experiments could be performed to identify the favorite dispensing method although the dispensing method would change the perception of the shape. The combination of dispensing methods and shape could be tested using Kansei Quantification Theory Type I [7].

Furthermore, during the focus group phases of the study, the participants often expressed how, when making the initial purchase decision, the functionality and ease of use at home would affect the decision. This highlights the difficulty in attempting to separate the factors that affect initial purchase and repurchase decisions. People have had experience of almost every product they purchase before they purchase it themselves.

The use of PrEmo in the validation tests compared well with the results of the semantic questionnaire. Participants thought it allowed them to express their feelings readily - it held their concentration better and was generally more interesting than semantic questionnaires. The participants felt that the threepoint scale was adequate although some would have liked the inclusion of an indifference option. As PrEmo uses predetermined emotions, and adjectives such as smooth, silky, or relaxing cannot be used, it might best be used for assessing the final stages of a design.

\section{Conclusions}

This article presented a case study in the elicitation and embodiment of affective requirements for moisturizer packaging. The case study is rigorous and comprehensive: the methodology used illustrates the stages of an ideal process that maps well onto the human-centered design framework. The methodology, 
based on the semantic differential technique, works well, but research challenges remain. The study highlights research issues associated with recombining stimuli that have been tested separately.

\section{Acknowledgments}

This study was carried out by Rebecca Livesey, Jeremy Westcott, Ben Parry, and John Lewis. The authors thank the Faraday Packaging Partnership for promoting their work in the packaging industry.

\section{References}

1. Kotonya, G. and Sommerville, I. (1998). Requirements Engineering Process and Techniques, John Wiley \& Son, Inc, Chichester.

2. Quality Systems \& Software, Inc./Telelogic, 200 Valley Road, Suite 306, Mt Arlington, NJ 07856, USA.

3. Hauser, J. and Clausing, D. (1988). The House of Quality, Harvard Business Review, 66: 63-73.

4. Jordan, P.W. (2000). Designing Pleasurable Products: An Introduction to the New Human Factors, Taylor \& Francis, London, New York.

5. Barnes, C.J., Childs, T.H.C., Henson, B. and Southee, C.H. (2004). Surface Finish and Touch - a Case Study in a New Human Factors Tribology, Wear, 257: 740-750.

6. Nagamachi, M. (2002). Kansei Engineering as a Powerful Consumer-oriented Technology for Product Development, Applied Ergonomics, 33(3): 289-294.

7. Schutte, S. (2002). Designing Feelings Into Products Integrating Kansei Methodology in Product Development, Linkoping University, Linkoping Studies in Science and Technology, Thesis No. 946.

8. Wright, P.C., McCarthy, J.C. and Meekison, L. (2003). Making Sense of Experience. In: Blythe, M., Monk, A., Overbeeke, C. and Wright, P.C. (eds), Funology: From Usability to User Enjoyment, pp. 43-53. Dordrecht: Kluwer.

9. Bruseberg, A. and McDonagh-Philip, D. (2001). New Product Development by Eliciting User Experience and Aspirations, International Journal of Human-computer Studies, 55: 435-452.

10. Preece, J., Rogers, Y. and Sharp, H. (2002). Interaction Design: Beyond Human-Computer Interaction, Wiley, New York, Chichester.

11. McQuaid, H.L., Goel, A. and McManus, M. (2003). When You Can't Talk To Customers: Using Storyboards and Narratives to Elicit Empathy For Users, DPPI'03, Pittsburgh, 23-26 June 2003.

12. Krippendorff (1989). Product Semantics; A Triangulation And Four Design Methods, In: Proceedings from the Product Semantics '89 Conference at the University of Industrial Arts, Helsinki, UIAH, 16-19 May 1989.

13. BS EN ISO 13407:1999 Human-centred design processes for interactive systems, British Standards Institution, London W4 4AL, UK, ISBN: 0580326187.

14. Osgood, C., Suci, G. and Tannenbaum, P. (1957). The Measurement of Meaning, Urbana: University of Illinois Press.
15. Krueger, R. and Casey, M. (2000). Focus Groups, 3rd edn, London: Sage.

16. Heneson, M., Morris, L. and Fitz-Gibbon, C. (1987). How to Measure Attitudes, 2nd edn, London: Sage.

17. Litwin, M. (1995). How to Measure Survey Reliability and Validity, London: Sage.

18. Munsell, A.H. (1971). A Color Notation: An Illustrated System Defining all Colors and their Relations by Measured Scales of Hue, Value, and Chroma, Munsell Color Company.

19. Suedfeld, P. (1990). Torture: A Brief Overview, In: Suedfeld, P. (ed.), Psychology and Torture, USA Hemisphere Publishing Corporation.

20. Bryant and Yarnold (1995). Principal Components Analysis and Exploratory and Confirmatory Factor Analysis, In: Grimm and Yarnold, Reading and Understanding Multivariate Statistics, American Psychological Association Books, Washington, D.C.

21. Desmet, P. (2003). Measuring Emotions: Development and Application of an Instrument to Measure Emotional Responses to Products. In Blythe, M., Monk, A., Overbeeke, C. and Wright P. C. (eds), Funology: From Usability to User Enjoyment. Dordrecht: Kluwer.

22. Bloj, M.G., Kersten, D. and Hurlbert, A. (1999). Perception of Three-dimensional Shape Influences Color Perception Through Mutual Illumination, Journal of Nature, 402: 877-879.

23. Guest, S. and Spence, C. (2003). What Role Does Mulitsensory Integration Play in the Visuotactile Perception of Texture?, International Journal of Psychophysiology, 50: 63-80.

24. Birren, F. (1978). Colour and Human Response: Aspects of Light and Colour Bearing on the Reactions of Living Things and the Welfare of Human Beings, New York: Van Nostrand Reinhold Company.

\section{Brian Henson}

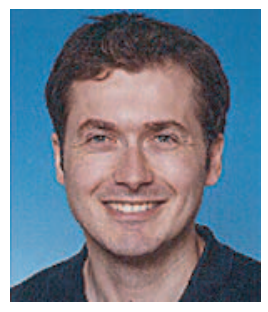

Brian Henson is a lecturer in Design and Manufacture in the School of Mechanical Engineering, University of Leeds. His principal research interests are in the areas of affective design and human-centered design of rehabilitation equipment. Within affective engineering he is particularly interested in multivariate analysis techniques and methods for measuring subjective responses to stimuli.

\section{Dr Cathy Barnes}

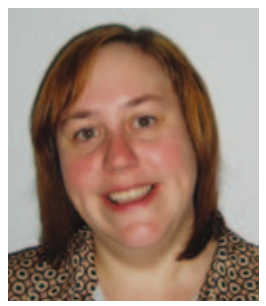

Dr Cathy Barnes is a lecturer in Design and Manufacture Integration in the School of Mechanical Engineering at the University of Leeds. Her research interests focus on the human interface of design and manufacturing and include affective design, 
emotional tribology and decision-based concurrent engineering and she has over 25 publications in these areas. She is leading the development of Kansei Engineering tools in a funded collaboration with nine major consumer goods companies. She has particular experience of experimental design, especially self-report elicitation of user feelings about products.

\section{Rebecca Livesey}

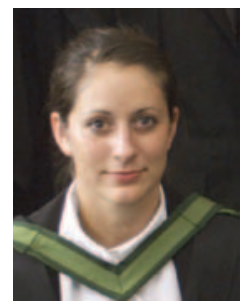

Rebecca Livesey graduated from the University of Leeds in 2004 with a first class honours degree in Management with Mechanical and Manufacturing Engineering. She is pursuing a career in the British army.

\section{Tom Childs}

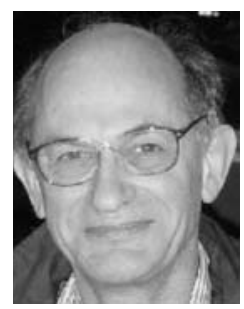

Tom Childs is Professor of Manufacturing Engineering in the School of Mechanical Engineering at the University of Leeds. His research interests include tribology and manufacturing process improvement. Recently the latter that has led to developing, with colleagues, a new activity at Leeds - Affective Engineering - the integration of engineering design, industrial design, and human factors research, to support the rapid development of products with intended affective and brand impact. $\mathrm{He}$ is currently a workpackage leader in the EU project ENGAGE and principal investigator of the UK EPSRC White Rose network for Affective Communication in Consumer Product and Exhibition Design.

\section{Keith Ewart} has been concerned with rapid prototyping processes. It is this

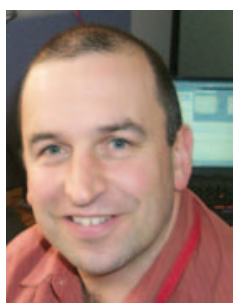

Keith Ewart works for Cre8Innov8, a small Corporate Packaging organization within Procter \& Gamble, which helps the business units to provide superior, creative, technology-driven ideas to win with customers, shoppers, and consumers. Keith leads Ideation processes and understanding of consumers' and shoppers' needs within this organization. He has worked for 15 years in Procter \& Gamble with particular experience in Cosmetics, Skin Care, and Corporate. In his social life Keith likes to spend time with his two young children (Calum, 6 and Ella, 4). In the little spare time he has left he can be found hacking his way around on the golf course. 